\title{
50 Years of Carbon Fibre
}

\author{
Peter Beaumont ${ }^{1}$
}

Received: 12 January 2017 / Accepted: 13 January 2017 / Published online: 18 February 2017

(C) Springer Science+Business Media Dordrecht 2017

With advances in computer power we understand the science of composites materials and composite technology in entirely new ways. It is possible to see the entire life cycle of the composite from its conception to its design and application across a broad spectrum of structural size: from the very small to the very large; from the processing of the material and manufacture of its parts and components; from the earliest stages of testing and characterization; and finally to its ultimate destruction in evaluating its reliability and durability thereby defining its limits of safe performance and structural integrity. We see how new techniques and methods of modeling and analysis have lead to a plethora of novel applications to meet society's needs.

To begin, it may have escaped the notice of the casual observer and user of carbon fiber products, such as the tennis racket or golf club, that carbon fiber has been around now for 50 years from those heady days of the 1960s when, as a matchbox curiosity, the short, black, "prickly" fiber was worth more than its weight in gold. Within a year, the fiber was being produced as continuous fiber in bundles or tows of 10,000 individual filaments wound onto spools that could be thrown like an American football to the back of college lecture theaters, fiber unravelling as it sped from the speaker's hand over heads of excited engineering students.

The discovery of this high strength, high stiffness, light-weight material that could be woven into fabric had returned us to those weaving factories of northern British towns of the 18th and 19th centuries. With fiber in and product out through the factory front door, we have arrived where material processing and manufacture, design and fabrication can now take place under one roof. We have arrived back at the factory of the future.

It is fitting on the occasion of half a century of carbon fiber to place on record the historical stages of the development of structural fiber composites, as well as premonitory things that happened on the way. An impressionistic map, if you like, of the present state of the art for readers new to the field as well as those ensconced in research and development of composite

Peter Beaumont

pwb1000@hermes.cam.ac.uk

1 University of Cambridge, Cambridge, Cambridgeshire, UK 
materials and large composite structures, from the emergence of the science of composites and the evolution of a number of neighbouring disciplines.

In the blink of an eye we have in composite materials discovered a clearly defined and distinct discipline, which in practice doubles up as a multi-discipline with a substantial number of independent branches, each one with its multifarious journals and textbooks, read by practicing materials scientists and engineers alike who communicate with each other on that basis. The discipline of materials science and engineering of contemporary composite materials has emerged from those small beginnings one half century ago.

Before carbon fiber, there was little materials science knowledge relating structure to properties of a composite material, although a wealth of knowledge of the physical metallurgy of metals and the chemistry of polymers and ceramics existed. We knew, for example, that covalent solids like alumina and silicon carbide were stiff and of low density, with a high melting point and having small coefficients of thermal expansion and thermal conductivity because of the spatial separation of their atoms by directed bonds. But high strength and ductility were considered mutually exclusive and a material that lacked ductility was thought of as being brittle and not worthwhile in an engineering context. In the search for stronger materials, there had been the discovery of a number of strong whisker crystals. The problem, however, associated with growing, harvesting and arranging them in a metal seemed insurmountable.

In the swinging sixties as the decade became known in the UK, a young professor at Cambridge, Alan Cottrell, often gave invited lectures at The Royal Society in London. In a discourse to The Royal Society Professor Cottrell cogently argued a novel treatment of a longstanding problem, namely the crack with a force between its faces. His direct approach to finding a solution was to obtain general expressions for the force and displacement between the crack surfaces.

On 15th June 1960, Professor Cottrell enunciated as follows:

“...No, the practical approach is to admit the existence of cracks and notches and to try to render them innocuous. If there is a transverse notch cutting across a parallel array of fibers in a rod of some material like adhesive, the forces from the cut fibers can be transmitted to the intact fibers close to the notch tip only by passing as shearing forces through layers of the adhesive." He continued: "If this adhesive has a fairly low resistance to shear... it will then be incapable of focussing the transmitted forces sharply... There is a tremendous opportunity for developing this principle further using fibers of very strong atomic forces like oxides and carbides."

Without necessarily realising it at the time, Cottrell had stated the principle of fiber reinforcement. Basically, we require in the design of a damage-tolerant composite material the presence of a microscopically weak structure built into a macroscopically strong solid that ensures any crack present becomes benign. Here, indeed, was a very striking phenomenon, ductility in a non-ductile material system. To produce ductility with a metal matrix is commonplace, but to produce it in a totally brittle system, that is something special that a crack could not extend if faced with an interface which yielded easily in shear.

A method of dissipating energy in composites, what the mechanicians call resistance to cracking, which commonly is called toughness, is vital in understanding the materials science of composites, which is otherwise an engineering discipline. Thus, the fragility of a brittle material could now be overcome by the incorporation of brittle fibers of oxides or carbides, for instance. By these means are engineering ceramics and brittle resins toughened. The key that 
unlocked the secret of toughened ceramics (and the adhesives) had been found, whether by laminating or by planar matting, or of oxide layers on metal surfaces, or of protective coatings, and it is of the utmost importance. Controlling the phenomena of micro-cracking in composites determines whether or not glasses or ceramics are to be used in gas turbine engines or carbon fiber and hybrid laminates of the fuselage and wings of the new generation of large commercial jet airliners, Boeing's Dreamliner (787) and Airbus's A380 and A350.

In an increasing number of textbooks and journals such as Applied Composite Materials, authors have collectively produced an impressionistic map of the present state of the science and mechanics of composite materials, seen as a pointillistic portrait of the discipline of composites, to be viewed from a slight distance. But what defines composite materials science and links it to traditional engineering disciplines? Perhaps the way to address this question is by means of what philosophers call an ostensive definition, relying on analogical or case-based reasoning. In other words, papers published over 5 decades are an essay in ostensive definition albeit a comprehensive one.

Throughout the volumes of this Journal we observe the materials scientist and engineer working at several levels of organization, each of which is underpinned by the next level. This feature is central to the subject of composite materials - the concept of architecture as the defining theme that connects composite materials science and engineering across orders of magnitude of size. At some point on this scale can be defined the concept of meso-structure, a term frequently used by modellers and simulators of composites, that level between the microscopic level and the macroscopic appearance from the viewpoint of the engineer.

Papers delve into the natural characteristics and behavior of various classes of engineering material reinforced in some way by fibre woven into high performance fabrics, probing and examining such concepts and relationships as structural architecture and design from the very small to the very large. What emerges is the evolution of a number of neighboring disciplines in mechanical design and processing: experimentation and analysis; mathematical and continuum modelling; constitutive and physical modelling (or micro-mechanics or damage mechanics); computational mechanics and virtual simulation aided by computer power.

Fine-scale phenomena become embedded in calculations representing larger-scale behaviour, arriving at intelligent mechanical design based upon the application of the principles of integrated multi-scale mechanics and hierarchical models and analyses. They all share certain characteristics in terms of hierarchy in which material constitutive properties are passed on from one model to the next via inheritance throughout the complete manufacture and design process.

Within the pages of this Journal, major themes range from descriptions and analyses of nano- and micro-scale phenomena that affect composite material behaviour at the metre level of size and above. They include the nature of cracks that nucleate and grow to threaten the safety of large engineering composite structures; fundamental material issues that affect composite weakness and compromise composite strength; and solutions to challenging material problems that hinder the safe exploitation of large composite structures. This requires an in-depth knowledge and understanding of the deformation and cracking processes in composites best detected and monitored directly, tracing damaging mechanisms and the progression of structural change over time. 
Materials engineers have focused on such topics as high toughness and impact resistance, fatigue damage progression, and environmental cracking. These research efforts have led to remarkable claims of stronger materials and scientific understanding but have not yet contributed to significant commercial exploitation across a wide spectrum of industrial sectors. Even now, basic design codes do not use the toughness of a composite or the growth-rate of fatigue cracks as failure criteria.

Standardization of composite materials, testing, decision-making procedures and other areas will also advance more rapidly in response to cost-cutting measures. The driving force for the application of composites in new technologies varies between industrial sectors and includes the capability for parts integration allowing savings in assembly costs, and lowercost tooling and rapid product change. The ability to achieve multifunctional properties is also important. Key factors in the choice of an appropriate manufacturing route include: speed and ease of product manufacture, properties and cost appropriate to purpose, potentially high-volume markets and the ability to increase automation for reduced costs and improved quality.

An area which is still not appreciated is the influence of fiber distribution and homogeneity: on surface appearance of the product; on methods for characterizing fiber distribution; and on properties such as toughness and the effect of notches, holes, and cut-outs on the fracture stress of the composite. Here lies a paradox at the heart of the design process of the very strong composite: the higher the intrinsic strength, the more subject the solid is to premature fracturing by crack propagation. The way forward, of course, is to obey Cottrell's hypothesis: to embed an ultra-strong ceramic or graphite in fibrous form in a relatively soft matrix of metal or polymer, more recently also of ceramic or graphite.

Today most structural composite systems are macroscopically elastic and fail under monotonic loading with negligible inelastic strain. Only appreciable inelastic strains develop within microscopic zones at or near to crack tips, or around notches or holes. The important consequence (particularly relevant for reinforced ceramics) is that stress redistribution micro-cracking mechanisms (matrix cracks; de-lamination cracks; splits; fiber pull-outs) exist around stress concentrators, such as notches. Consequently, the stress peaks are quite small and diffuse, such that the survival probability of the composite can be determined by comparing the peak stresses within the fiber bundle strength. The composite dilates with enhanced compliance and stress concentrations diminish substantially below their elastic values. In some cases, the composite is totally notch insensitive, and the stress concentrations at the notch are eliminated by matrix microcracking and fiber pull-out. The important consequence is that the design problem has a better definition. Thus, the composite of low density and near-net-shape fabricability, as well as manufacturing robustness is provided with remarkable damage tolerance. We have arrived at the ideal definition of an engineering composite resembling the strong solid as postulated by Cottrell.

Our technology foresight has brought together in the pages of this Journal the academic and industrial experts to formulate the targets for new and improved materials, a rationalization of fiber product ranges and the use of higher tex yarns in unidirectional and woven fabric-based products. Another area of material development includes biomimetic materials having the ability to repair themselves or be induced to self-heal implants that last the life of the patient.

But that which weaves the threads of journal pages is an understanding of the structural integrity of the composite. By means of careful experimentation and sound analysis, this 
ultimately leads to successful forecasting of the limits of material performance on the one hand and defines the conditions for safe operation of composite structure on the other hand from "cradle to grave".

Robert Hooke writes in his book Micrographia (1665), an imprint of The Royal Society of London:

And I have often thought that probably there might be a way found out to make an artificial glutinous composition much resembling if not full as good, nay better than that excrement or whatever substance it might be out of which the silk worm draws his clew. If such a composition were found it were certainly an easy matter to find very quick ways of drawing it into small wires ${ }^{*}$ for use. I do not mention the use of such an invention nor the benefit that is likely to accrue to the finder, they being fully obvious. This hint may I therefore hope to give some inquisitive person an occasion of making some trials which if successful I have my aim and I suppose he will have no occasion to be displeased.

('Old English wīr; Germanic origin, probably the base of Latin viere plait, weave).

Peter Beaumont (Editor)

Cambridge 